\title{
Resiliencia en gestantes adolescentes del Caribe colombiano: evaluación con la escala de Wagnild y Young
}

\author{
Álvaro Monterrosa-Castro ${ }^{1}$, Liezel Ulloque-Caamaño² ${ }^{2}$ Candelaria Colón-Iriarte³ , Ester Polo-Payares ${ }^{4}$
}

\section{RESUMEN}

Introducción: las adolescentes presentan cambios biológicos y reciben influencias sociales que las predisponen a quedar embarazadas a temprana edad.

Objetivo: evaluar la resiliencia en un grupo de gestante adolescentes para estimar la frecuencia deI NRB (nivel de resiliencia bajo) e identificar factores psicosociales asociados.

Metodología: estudio transversal en gestantes adolescentes que acudieron a consulta prenatal en Cartagena, Colombia. Se aplicó un cuestionario de características sociodemográficas y escalas sobre resiliencia, familismo, religiosidad, espiritualidad, funcionalidad familiar, violencia de pareja, felicidad y autoestima. Se compararon dos grupos: NRB y NRAM (nivel de resiliencia alto/moderado). Se realizó una regresión logística multivariada para identificar los factores asociados al NRB.

Resultados: se estudiaron 499 gestantes adolescentes con una edad promedio de 17 años. La mitad de ellas pertenecían a familias disfuncionales. Sesenta y cuatro $(12,8 \%)$ tuvieron un NRB con factores asociados como bajo familismo OR: 4,736 (IC $95 \%$ : 2,673-8,392), baja autoestima OR: 4,257 (IC $95 \%$ : 1,363-13,295), disfunción familiar OR: 3,902 (IC 95 \%: 2,052-7,422), violencia de pareja OR: 2,689 (IC 95 \%: 1,082-6,678), deficiente espiritualidad OR: 2,503 (IC 95 \%: 1,4614,287 ) y reducida felicidad OR: 2,063 (IC $95 \%: 1,214-3,506$ ).

1 Médico. Especialista en ginecología y obstetricia. Profesor. Líder del grupo de investigación salud de la mujer. Facultad de Medicina. Universidad de Cartagena, Colombia.

Médico. Magister en epidemiología. Integrante del grupo de investigación salud de la mujer. Facultad de Medicina. Universidad de Cartagena, Colombia.

3 Enfermera. Magister en estudios de género, área mujer y desarrollo. Profesora. Integrante del grupo de investigación salud, mujer e infancia. Facultad de Enfermería. Universidad de Cartagena, Colombia.

4 Enfermera. Magister en estudios de género, área mujer y desarrollo. Profesora. Integrante del grupo de investigación salud, mujer e infancia. Facultad de Enfermería. Universidad de Cartagena, Colombia.

Correspondencia: Álvaro Monterrosa-Castro, alvaromonterrosa@gmail.com

Recibido: septiembre 3 de 2019

Aceptado: noviembre 25 de 2019

Cómo citar: Monterrosa-Castro A, Ulloque-Caamaño L, Colón-Iriarte C, Polo-Payares E. Resiliencia en gestantes adolescentes del Caribe colombiano: evaluación con la escala de Wagnild y Young. latreia. 2020 Jul-Sep;33(3):209-21. DOI 10.17533/udea.iatreia.51. 
Conclusión: en una ciudad del Caribe colombiano, en trece de cada cien gestantes adolescentes que acuden a consulta prenatal, se encontró NRB; disfunción familiar, violencia de pareja en el embarazo, deficiente perspectiva espiritual, reducida felicidad subjetiva, familismo y nivel de autoestima bajos se observaron asociados.

\section{PALABRAS CLAVE}

Embarazo en Adolescencia; Factores de Riesoo; ResiIiencia Psicolóóoica; Servicios de Salud del Adolescente

\section{SUMMARY}

Resilience in pregnant adolescents of the Colombian Caribbean, evaluation with the Wagnild and Young scale

Introduction: Adolescents present biological changes and receive social influences which predispose them to pregnancies at early age.

Objective: To evaluate resilience in a group of pregnant adolescents, to estimate the frequency of low resilience level and identify associated psychosocial factors.

Methodology: Cross-sectional study in pregnant adolescents who went to prenatal visit in Cartagena, Colombia. A sociodemographic characteristics questionnaire and validated scales on resilience, familism, religiosity, spirituality, family functionality, partner violence, happiness and self-esteem were applied. Two groups were compared: high/moderate resilience level (HMRL) and low resilience level (LRL). Multivariable logistic regression was performed to identify factors associated with LRL.

Results: 499 pregnant adolescents were studied, average age 17 years, half belonging to dysfunctional families. Sixty four $(12,8 \%)$ had LRL and were associated factors: low familism OR: 4,736 (IC95\%: 2,673-8,392), low self-esteem OR: 4,257 (IC95\%: 1,363-13,295), family dysfunction OR: 3,902 (IC95\%: 2,052-7,422), partner violence OR:2,689 (IC95\%: 1,082-6,678), poor spirituality OR: 2,503 (IC95\%: 1,461-4,287) and reduced happiness OR: 2,063 (IC95\%: 1,214-3,506).
Conclusion: In a city of the Colombian Caribbean, in thirteen of every one hundred pregnant adolescents who attend to prenatal visit, LRL was found. Family dysfunction, partner violence in pregnancy, poor spirituality, reduced subjective happiness, low familism and low level of self-esteem, were associated with LRL.

\section{KEY WORDS}

Adolescent Health Services; Pregnancy in Adolescence; Resilience, Psychological; Risk Factors

\section{INTRODUCCIÓN}

La adolescencia, etapa vital entre los 10 y 19 años, se subdivide en temprana (10-14 años) y tardía (15-19 años) ${ }^{(1)}$. En ella se presentan retos y situaciones sociales, educativas y personales que pueden hacer que las adolescentes estén en condiciones de vulnerabilidad, expuestas, especialmente, a un embarazo temprano $^{(1,2)}$.

Según la OMS, dieciséis millones de mujeres entre los 15 y 19 años y, aproximadamente, un millón de niñas menores de 15 años, tienen trabajos de parto al año (2). La más reciente encuesta de demografía y salud de Colombia señaló que la tasa de fecundidad para este país fue de 74 nacidos vivos por cada mil adolescentes en el año $2015^{(3)}$.

En general, el embarazo en menores de 19 años suele ser considerado de alto riesgo ${ }^{(1,2,4,5)}$, no obstante, no solo los aspectos médicos se deben evaluar. Se ha indicado que las gestantes adolescentes, comparadas con las adultas, pueden experimentar mayores problemas psicosociales, especialmente la interrupción de la educación, el desempleo, aislamiento, violencia de pareja, deterioro del bienestar y un pobre desarrollo social ${ }^{(1,2,5-7)}$. El embarazo en la adolescencia debe ser visto como una experiencia adversa que trunca el accionar futuro, pues contribuye a que las jóvenes renuncien a la construcción de sus planes de desarrollo y proyectos de vida que generan una superación personal ${ }^{(3,4,6,8,9)}$. El embarazo en la adolescencia debe ser prevenido, multidisciplinariamente enfrentado $y$ atendido por profesionales de la salud 
suficientemente capacitados ${ }^{(10,11)}$. Salazar et al. ${ }^{(5)}$ han informado que las embarazadas adolescentes suelen tener menores niveles de resiliencia.

La resiliencia es la capacidad que poseen las personas para recuperarse, readquirir fuerzas, continuar la vida $y$ proyectarse al futuro a pesar de las adversidades ${ }^{(5)}$. Esta constituye un proceso dinámico influenciado por factores psicosociales: creencias religiosas, apoyo social, nivel de escolaridad, edad, pobreza, ocupación, familia, consumo de alcohol o psicoactivos, autoestima y percepción de la felicidad, entre $\operatorname{otros}^{(4,5,12-14)}$.

Usualmente, la mayoría de los estudios sobre el embarazo en adolescentes abordan la problemática solo desde lo obstétrico ${ }^{(1,2,15)}$. Las implicaciones psicosociales del embarazo en la adolescencia han sido descritas ampliamente, pero escasamente la resiliencia u otras psicopatologías ${ }^{(5)}$. La propuesta de las Naciones Unidas, denominada Estrategia Mundial para Ia SaIud de la Mujer, el Niño y el Adolescente (2016-2030), enfatiza en que hoy es más importante que nunca, para fomentar el crecimiento económico y disponer de una población sana, crear comunidades que sean resilientes en diversos tipos de choques ${ }^{(8)}$. Por tanto, para ser coherentes y generar planes de acción, es necesario estimar cifras e indicadores. Por ello, el objetivo de este estudio fue evaluar la resiliencia en las gestantes adolescentes y así poder estimar la frecuencia del nivel de resiliencia bajo e identificar su asociación con otros factores psicosociales.

\section{METODOLOGÍA}

\section{Diseño}

Estudio observacional transversal, con análisis anidado de casos y controles, realizado en gestantes adolescentes de la consulta ambulatoria de la E.S.E. Cartagena de Indias y la Maternidad Rafael Calvo, instituciones públicas de atención en salud de la ciudad Cartagena, región del Caribe colombiano. El estudio hace parte de la línea de investigación: Gestación. Para la toma de información se utilizó la entrevista y el auto diligenciamiento.

\section{Participantes}

Se incluyeron mujeres con edades entre los 10 y 19 años con embarazo de más de ocho semanas de gestación, que no tuviesen situación de emergencia en esa consulta $y$ acudiesen al control prenatal con un acompañante mayor de edad (18 años o más, según la legislación colombiana). Fueron invitados a participar por médicas o enfermeras previamente capacitadas, quienes les explicaron los alcances del estudio. Se excluyeron las que no desearon participar, no fueron autorizadas por el acompañante o tenían limitaciones síquicas, físicas o de lectoescritura que les impidiera diligenciar el instrumento. Las gestantes podían suspender el llenado del formulario $y$ retirarse.

\section{Herramientas}

Se aplicó un formulario que contenía dos partes. La primera era un cuestionario con las preguntas sociodemográficas (historia clínica ginecológica y obstétrica, hábitos, costumbres, datos del compañero sexual y de la familia) que la gestante contestó a la encuestadora. La segunda, consistió en la aplicación de las siguientes escalas.

1) Escala de resiliencia de Wagnild y Young: identifica cualidades personales que favorecen la adaptación individual resiliente $y$ permite conocer la personalidad positiva. Consta de 25 ítems que se califican desde 1 (no estar de acuerdo) hasta 7 (estar de acuerdo). A mayor puntuación mayor resiliencia, además, establece tres niveles: alto (147 o más puntos), moderado $(146-121)$ y bajo (menos de 121) ${ }^{(16)}$.

2) Escala de perspectiva espiritual. Mide dicha visión en la persona y las interacciones con la religiosidad. Son diez enunciados, los cuatro primeros se responden desde 1 (nunca) hasta 6 (más o menos una vez al día) y, las restantes, desde 1 (en desacuerdo) hasta 6 (de acuerdo). A mayor puntaje mayor espiritualidad (17). Este concepto hace referencia a los sentimientos, pensamientos, experiencias y comportamientos que surgen de la búsqueda de lo sagrado; estimula, motiva y permite la búsqueda de trascendencia, propósitos $y$ sentido para vivir ${ }^{(17-19)}$.

3) Escala de familismo de Bardis. Mide el familismo según el grado de acuerdo o desacuerdo ante afirmaciones que implican compromiso, disposición a ayudar, a obedecer o estar cerca de la familia. Cada ítem tiene una puntuación de 0-5, a mayor puntuación mayor familismo ${ }^{(20)}$. El término familismo se refiere a 
la identificación y apego que tienen las personas con sus familias, ya sean nucleares o extensas ${ }^{(4)}$.

4) EI APGAR familiar determina la funcionalidad familiar. Los puntajes de cada respuesta van desde el 0 (nunca) hasta 4 (siempre): de 18-20 puntos indica una función familiar normal $y$, menos de 18, refiere una disfunción familiar ${ }^{(21)}$.

5) Abuse Assessment Screen identifica la violencia de pareja con cinco preguntas que deben ser respondidas: "Sí" o "No". Una respuesta positiva en los ítems 2, 3 y 4 indica una víctima de violencia de pareja. ${ }^{(22)}$. La OMS define la violencia de pareja como el comportamiento dentro de una relación íntima que causa daño físico, sexual o psicológico; incluye el comportamiento de control ${ }^{(2,4,9)}$. Por el contexto del estudio, se interrogó específicamente sobre violencia de pareja en el embarazo.

6) Escala de felicidad, valora el estado de felicidad con cuatro ítems que se califican desde 1 (menos feliz) hasta 7 (más feliz). A mayor puntuación mayor felicidad ${ }^{(23)}$. La felicidad es un concepto multidimensional que coincide con el objetivo de las personas, pues es el balance de lo positivo y negativo. Se ha definido como el estado afectivo de satisfacción que experimenta el individuo en posesión del bien anhelado ${ }^{(4)}$.

7). Escala de autoestima de Rosenberg, compuesta por diez afirmaciones, cinco redactadas positiva y cinco negativas. Se evalúa desde 1 (desacuerdo) hasta 4 (de acuerdo). A mayor puntuación mayor autoestima, se considera normal los 25 o más puntos ${ }^{(24)}$.

\section{Tamaño de Ia muestra}

Fue estimado mediante el software Epidat (versión 3.01) basado en una población elegible de 6.428 adolescentes embarazadas, con una prevalencia del nivel de resiliencia bajo 34,2 \% según el hallazgo en una población similar ${ }^{(9)}$, un nivel de confianza del $95 \%$ y error de muestreo del $5 \%$. Se calculó en 494 el tamaño de la muestra. Se agregaron 49 (10 \%) formatos para compensar los invalidados, por lo tanto, se tuvieron disponibles 543 formularios que se aplicaron diariamente. Se llevó el conteo de las adolescentes excluidas del estudio.

\section{Análisis estadístico}

Se realizó con Epi Info 7. Los datos categóricos se expresan en frecuencias absolutas $y$ en porcentajes con intervalos de confianza del $95 \%$. A las variables cuantitativas se les probó la distribución de los datos, mediante la prueba de normalidad KolmogorovSmirnov; se observó que tenían distribución no paramétrica. Se conformaron dos grupos: NRAM (nivel de resiliencia alto/moderado) y NRB (nivel de resiliencia bajo). Las diferencias de las medianas o medias fueron estimadas con ANOVA o Kruskal-Wallis, según la diferencia de varianzas obtenida con la prueba de Bartlett. Las diferencias de los porcentajes se valoraron con chi cuadrado. Se adelantó regresión logística multivariada. El nivel de resiliencia fue la variable dependiente y las otras escalas fueron las variables independientes, dicotomizadas según el punto de corte (violencia de pareja, disfunción familiar $y$ autoestima) o el promedio encontrado (familismo, perspectiva espiritual y felicidad). También fueron variables independientes las características sociodemográficas. $\mathrm{p}<0,05$ fue considerada estadísticamente sionificativa.

\section{Ética}

Proyecto avalado por el comité de ética de la Universidad del Norte, de la Universidad de Cartagena y de las dos instituciones de atención. La participación de las gestantes fue voluntaria, firmaron el asentimiento informado y el acompañante firmó el consentimiento informado. Se tuvieron en cuenta las recomendaciones de la Declaración de Helsinki y de la Resolución 8430 de 1993 del Ministerio de Salud de Colombia, que clasifica el presente estudio como de riesgo mínimo.

\section{RESULTADOS}

Se invitaron a participar 565 gestantes adolescentes, $22(3,8 \%)$ no desearon participar o tuvieron criterios para su exclusión. Se aplicaron 543 formularios: 44 $(8,1 \%)$ estuvieron incorrectamente diligenciados y $499(91,9 \%)$ estaban completos $y$ adecuados para el estudio.

La Tabla 1 presenta las características sociodemográficas de las participantes y su distribución entre el NRAM o NRB. La mediana de edad fue de 17 años, la mitad de las participantes pertenecían a familias disfuncionales según el APGAR familiar; el 3 \% consideró no tener apoyo familiar para el embarazo o tenían baja autoestima, el $5 \%$ había sido víctima de violencia de pareja dentro del embarazo. Entre las gestantes 
adolescentes afrodescendientes hubo una mayor frecuencia del NRB en comparación con las mestizas, $31,2 \%$ vs. 12,2 \%, respectivamente. A su vez, las jóvenes con un NRB tuvieron una menor puntuación en perspectiva espiritual, familismo y felicidad que el que tenían las gestantes con un NRAM ( $<<0,05)$. La carencia del apoyo y la disfunción familiar, la violencia de pareja, presencia de patología en el embarazo y baja autoestima, fueron más frecuentes entre las adolescentes con un NRB ( $p<0,05)$. No se observaron diferencias en la edad, etapa de adolescencia, zona de residencia, estrato socioeconómico, escolaridad, ocupación laboral, asistencia a la iglesia, condición de religiosidad, semanas de gestación, trimestre de inicio del control prenatal, número de ecografías, embarazo de alto riesgo, personas que convivían en casa, consumo de cigarrillo, alcohol o psicoactivos, apoyo de amigos, acompañamiento de entidades gubernamentales, escolaridad, convivencia, apoyo y edad del compañero sexual ( $p>0,05)$. Tuvieron un NRB 3 (14,2 $\%)$ de las 21 gestantes que estaban en la adolescencia temprana y, 61 (12,7\%) de las 478 que estaban en la adolescencia tardía. $\mathrm{X}^{2}=0,041$ (Mantel-Haenszel), $\mathrm{p}=0,8$.

Tabla 1. Características sociodemográficas y su distribución según el nivel de resiliencia

\begin{tabular}{|c|c|c|c|c|}
\hline \multirow{3}{*}{ Variables } & Todas & NRAM $^{*}$ & NRB $^{+}$ & \multirow{3}{*}{$\mathbf{p}$} \\
\hline & $\begin{array}{c}499 \\
(100 \%)\end{array}$ & $\begin{array}{c}435 \\
(87,2 \%)\end{array}$ & $\begin{array}{c}64 \\
(12,8 \%)\end{array}$ & \\
\hline & & Me (RI) & & \\
\hline Edad en años & $17,0(2,0)$ & $17,0(2,0)$ & $17,0(3,0)$ & $0,927^{\ddagger}$ \\
\hline Puntuación escala perspectiva espiritual & $47,7(11,0)$ & $48,0(10,0)$ & $43,0(11,5)$ & $<0,001^{\ddagger}$ \\
\hline Puntuación escala de familismo & $43,0(9,0)$ & $44,0(9,0)$ & $36,0(15,0)$ & $<0,001^{\ddagger}$ \\
\hline \multirow[t]{2}{*}{ Puntuación escala de felicidad subjetiva } & $28,0(5,0)$ & $28,0(4,0)$ & $24,0(6,0)$ & $0,002^{\ddagger}$ \\
\hline & \multicolumn{3}{|c|}{ n (\%) (IC $95 \%)$} & \\
\hline Etapa temprana de la adolescencia & $\begin{array}{c}21(4,2) \\
(2,7-6,5)\end{array}$ & $\begin{array}{c}18(85,7) \\
(63,7-97,0)\end{array}$ & $\begin{array}{c}3(14,3) \\
(3,1-36,3)\end{array}$ & \multirow{2}{*}{$0,838^{\S}$} \\
\hline Etapa tardía de la adolescencia & $\begin{array}{l}478(95,8) \\
(93,5-97,3)\end{array}$ & $\begin{array}{c}417(87,2) \\
(83,8-90,0)\end{array}$ & $\begin{array}{c}61(12,8) \\
(10,0-17,2)\end{array}$ & \\
\hline Residencia en área urbana & $\begin{array}{c}362(72,5) \\
(68,4-76,4)\end{array}$ & $\begin{array}{l}319(88,1) \\
84,2-91,2)\end{array}$ & $\begin{array}{l}43(11,9) \\
(8,8-15,8)\end{array}$ & \multirow{2}{*}{$0,304^{\S}$} \\
\hline Residencia en el área rural & $\begin{array}{c}137(27,5) \\
(23,6-31-6)\end{array}$ & $\begin{array}{l}116(84,7) \\
77,5-90,3)\end{array}$ & $\begin{array}{l}21(15,3) \\
(9,8-22,5)\end{array}$ & \\
\hline En convivencia con el compañero sexual & $\begin{array}{c}427(85,6) \\
(82,1-88,5)\end{array}$ & $\begin{array}{l}373(87,4) \\
(83,7-90,3)\end{array}$ & $\begin{array}{l}54(12,6) \\
(9,7-16,3)\end{array}$ & \multirow{2}{*}{$0,771^{\S}$} \\
\hline Sin convivencia con el compañero sexual & $\begin{array}{c}72(14,4) \\
(11,5-17,9)\end{array}$ & $\begin{array}{c}62(86,1) \\
(75,9-93,1)\end{array}$ & $\begin{array}{c}10(13,9) \\
(6,9-24,1)\end{array}$ & \\
\hline Etnia mestiza & $\begin{array}{l}483(96,8) \\
(94,7-98,5)\end{array}$ & $\begin{array}{l}424(87,8) \\
(84,5-90,5)\end{array}$ & $\begin{array}{l}59(12,2) \\
(9,5-15,6)\end{array}$ & \multirow{2}{*}{$0,025^{\S}$} \\
\hline Etnia Afrodescendiente & $\begin{array}{c}16(3,2) \\
(1,9-5,2)\end{array}$ & $\begin{array}{c}11(68,8) \\
(41,3-89,0)\end{array}$ & $\begin{array}{c}5(31,2) \\
(11,0-58,7)\end{array}$ & \\
\hline
\end{tabular}

(continúa) 
Tabla 1. Características sociodemográficas y su distribución según el nivel de resiliencia (Continuación)

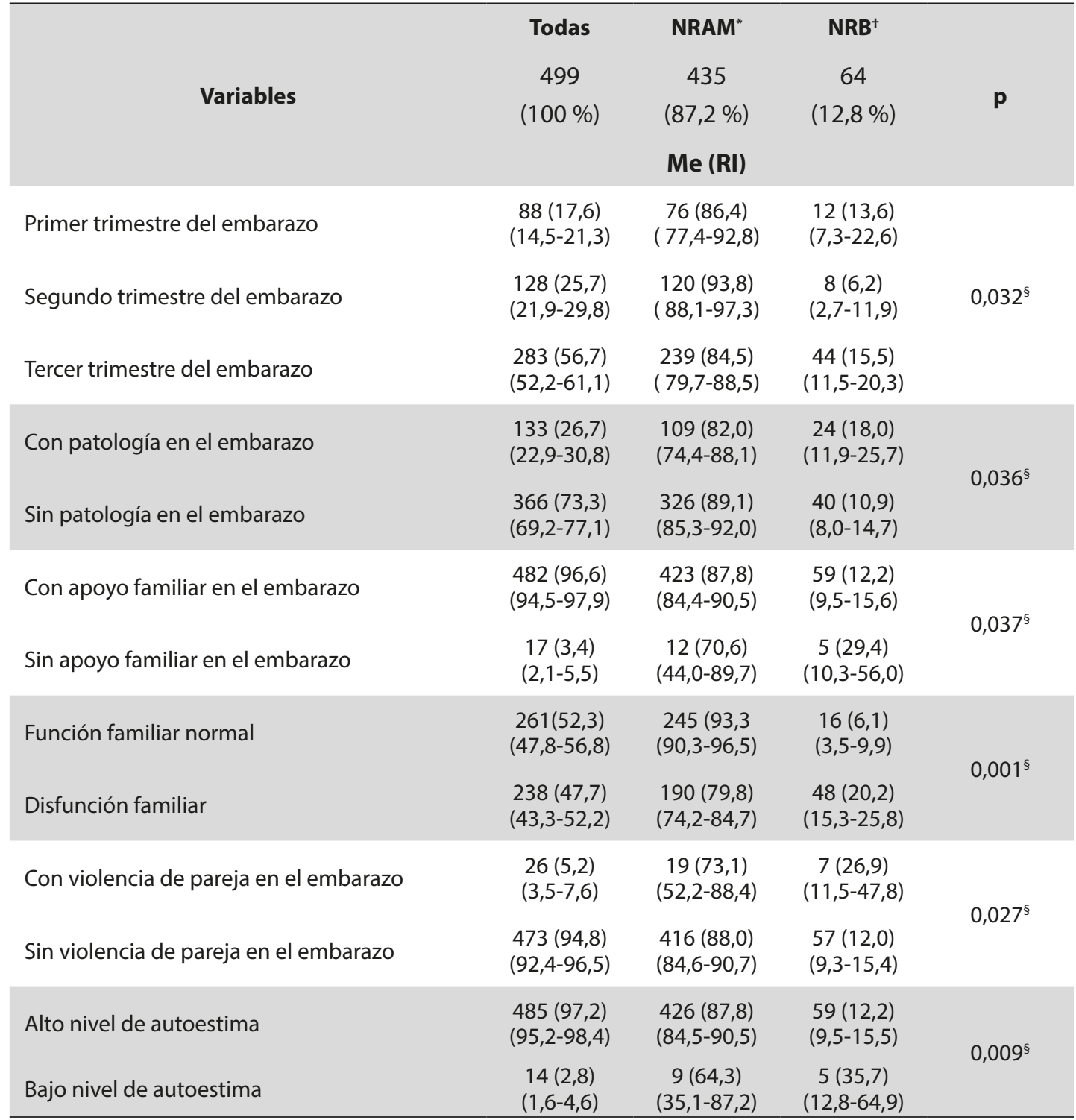

* NRAM: Nivel de resiliencia alto/moderado. † NRB: Nivel de resiliencia bajo. ‡ Test de Kruskal-Wallis. § Prueba de chi cuadrado. Fuente: cuestionario de características sociodemográficas, escala de perspectiva espiritual, escala de familismo de Bardis, escala de felicidad, APGAR familiar, violencia de pareja, autoestima y resiliencia

Se encontraron los siguientes niveles de resiliencia: alto 138 (27,7 \%) (IC $95 \%$ : 23,8-31,8), moderado 297 $(59,5 \%)$ (IC $95 \%: 55,1-63,8)$ y bajo $64(12,8 \%$ ) (IC $95 \%$ : 10,1-16,2). Se identificó el alfa de Cronbach: 0,747. La Tabla 2 presenta la media y desviación estándar de cada uno de los ítems de la escala. Aquellos que turieron una mayor puntuación: "Siento orgullo de haber logrado cosas en mi vida" y "Mi vida tiene sentido"; menor puntuación: "A veces yo hago cosas quiera o nwe preguntó sobre el objetivo de las cosas" y "No 
insisto en cosas en las que no puedo hacer nada al respecto". Las jóvenes con un NRAM tuvieron una mejor puntuación en todos los ítems que las que tuvieron un
NRB. La puntuación de la escala para todos los participantes: $136,9 \pm 20,7$; las que tuvieron un NRAM 142,8 \pm 11,1 y, las del grupo NRB: $96,4 \pm 25,4(p<0,001)$.

Tabla 2. Evaluación del nivel de resiliencia con la escala de Wagnild y Young

\begin{tabular}{|c|c|c|c|c|}
\hline \multirow{4}{*}{ Ítems } & Todas & NRAM $^{*}$ & $\mathbf{N R B}^{+}$ & \multirow{4}{*}{$\mathbf{P}$} \\
\hline & 499 & 435 & 64 & \\
\hline & $(100 \%)$ & $(87,2 \%)$ & $(12,8 \%)$ & \\
\hline & \multicolumn{3}{|c|}{ Media \pm desviación estándar } & \\
\hline Usualmente puede ver una situación desde varios puntos de vista & $5,3 \pm 1,4$ & $5,5 \pm 1,2$ & $3,9 \pm 1,9$ & $<0,001^{\ddagger}$ \\
\hline La confianza en mí misma me permite pasar los tiempos difíciles & $5,7 \pm 1,3$ & $6,0 \pm 1,0$ & $4,0 \pm 2,0$ & $<0,001^{\ddagger}$ \\
\hline Soy decidida & $5,8 \pm 1,3$ & $6,1 \pm 0,8$ & $3,9 \pm 1,9$ & $<0,001^{\ddagger}$ \\
\hline Usualmente manejo los problemas de distintos modos & $5,5 \pm 1,3$ & $6,1 \pm 0,8$ & $3,9 \pm 1,9$ & $<0,001^{\ddagger}$ \\
\hline Siento que puedo manejar varias cosas a la vez & $4,9 \pm 1,6$ & $5,2 \pm 1,5$ & $3,0 \pm 1,5$ & $<0,001^{\S}$ \\
\hline $\begin{array}{l}\text { Cuando estoy en una situación difícil generalmente encuentro } \\
\text { salida }\end{array}$ & $5,6 \pm 1,3$ & $5,8 \pm 1,0$ & $3,9 \pm 1,7$ & $<0,001^{\ddagger}$ \\
\hline $\begin{array}{l}\text { Soy capaz de hacer las cosas por mí misma sin depender de los } \\
\text { demás }\end{array}$ & $5,3 \pm 1,5$ & $5,6 \pm 1,2$ & $3,1 \pm 1,7$ & $<0,001^{\ddagger}$ \\
\hline Puedo encontrar, generalmente, algo sobre que reírme & $6,0 \pm 1,1$ & $6,3 \pm 0,6$ & $4,3 \pm 2,1$ & $<0,001^{\ddagger}$ \\
\hline $\begin{array}{l}\text { En una emergencia, soy alguien en quien las personas pueden } \\
\text { confiar }\end{array}$ & $5,9 \pm 1,2$ & $6,1 \pm 0,8$ & $4,5 \pm 1,9$ & $<0,001^{\ddagger}$ \\
\hline Siento orgullo de haber logrado cosas en mi vida & $6,1 \pm 1,0$ & $6,3 \pm 0,7$ & $4,9 \pm 2,0$ & $<0,001^{\ddagger}$ \\
\hline $\begin{array}{l}\text { He podido atravesar situaciones difíciles, porque he experimenta- } \\
\text { do dificultades antes }\end{array}$ & $5,6 \pm 1,4$ & $5,9 \pm 1,0$ & $3,4 \pm 2,0$ & $<0,001^{\ddagger}$ \\
\hline Me basto a mí misma si lo creo necesario & $5,5 \pm 1,3$ & $5,8 \pm 1,0$ & $3,3 \pm 1,8$ & $<0,001^{\ddagger}$ \\
\hline A veces yo hago cosas, quiera o no & $4,5 \pm 1,7$ & $4,7 \pm 1,7$ & $3,2 \pm 1,6$ & $<0,001^{\S}$ \\
\hline Mantengo interés por las cosas & $5,2 \pm 1,7$ & $5,5 \pm 1,5$ & $3,4 \pm 1,8$ & $<0,001^{\ddagger}$ \\
\hline Mi vida tiene sentido & $6,1 \pm 1,0$ & $6,3 \pm 0,6$ & $4,9 \pm 3,9$ & $<0,001^{\ddagger}$ \\
\hline $\begin{array}{l}\text { Generalmente tengo energía para hacer aquello que tengo que } \\
\text { hacer }\end{array}$ & $5,8 \pm 1,2$ & $6,0 \pm 1,0$ & $3,7 \pm 1,9$ & $<0,001^{\ddagger}$ \\
\hline Soy auto disciplinada & $5,3 \pm 1,5$ & $5,6 \pm 1,3$ & $3,9 \pm 1,8$ & $<0,001^{\ddagger}$ \\
\hline Mantengo el interés en aquellas cosas importantes para mí & $5,9 \pm 1,2$ & $6,2 \pm 0,9$ & $4,4 \pm 2,0$ & $<0,001^{\ddagger}$ \\
\hline Cuando hago planes los llevo a cabo hasta el final & $5,7 \pm 1,3$ & $5,9 \pm 1,0$ & $3,9 \pm 2,0$ & $<0,001^{\ddagger}$ \\
\hline Acostumbro a tomar las cosas sin mucha preocupación & $5,1 \pm 1,6$ & $5,4 \pm 1,4$ & $3,5 \pm 2,0$ & $<0,001^{\ddagger}$ \\
\hline Soy amigable conmigo misma & $6,0 \pm 1,1$ & $6,2 \pm 0,6$ & $4,5 \pm 2,1$ & $<0,001^{\ddagger}$ \\
\hline Hago las cosas de a una por vez & $5,2 \pm 1,5$ & $5,4 \pm 1,3$ & $3,4 \pm 1,9$ & $<0,001^{\ddagger}$ \\
\hline Rara vez me pregunto sobre el objetivo de las cosas & $4,8 \pm 1,6$ & $5,1 \pm 1,5$ & $3,2 \pm 1,5$ & $<0,001^{\S}$ \\
\hline No insisto en cosas en las que no puedo hacer nada al respecto & $4,7 \pm 1,7$ & $5,0 \pm 1,6$ & $3,0 \pm 1,7$ & $<0,001^{\S}$ \\
\hline Me siento cómoda si hay gente que no me agrada & $3,8 \pm 2,1$ & $3,9 \pm 2,1$ & $3,0 \pm 1,9$ & $0,001^{\S}$ \\
\hline
\end{tabular}

* NRAM: Nivel de resiliencia alto/moderado. † NRB: Nivel de resiliencia bajo. ‡ Test de Kruskal-Wallis. § Prueba de chi cuadrado. Fuente: escala de resiliencia de Wagnild y Young 
Se realizó la regresión logística ajustada, resultados en la Tabla 3. Seis factores psicosociales se asociaron con un NRB: bajo familismo, bajo nivel de autoestima, disfunción familiar, violencia de pareja en el embarazo, deficiente perspectiva espiritual y reducida felicidad subjetiva $(\mathrm{p}<0,05)$. Por cada punto que aumentaba la escala de perspectiva espiritual, familismo y felicidad, se reducía la puntuación de la escala de resiliencia en 0,049; 0,079 y 0,081, respectivamente. A medida que se disminuían las puntuaciones en cada una de esas escalas había una mayor presencia del NRB. El segundo trimestre del embarazo se asoció con un menor NRB, en referencia al tercer trimestre.

Tabla 3. Factores asociados con el nivel de resiliencia en la regresión logística ajustada. $n=499$

\begin{tabular}{|c|c|c|}
\hline Factores & OR (IC $95 \%)$ & $\mathbf{p}^{*}$ \\
\hline $\begin{array}{l}\text { Alto familismo } \\
\text { Bajo familismo }\end{array}$ & $\frac{1}{4,736(2,673-8,392)^{+}}$ & $<0,001$ \\
\hline $\begin{array}{l}\text { Alto nivel de autoestima } \\
\text { Bajo nivel de autoestima }\end{array}$ & $\begin{array}{c}1 \\
4,257(1,363-13,295)^{\ddagger}\end{array}$ & 0,013 \\
\hline $\begin{array}{l}\text { Funcionalidad familiar normal } \\
\text { Disfunción familiar }\end{array}$ & $\begin{array}{c}1 \\
3,902(2,052-7,422)^{\S}\end{array}$ & $<0,001$ \\
\hline $\begin{array}{l}\text { Ausencia de violencia de pareja en el embarazo } \\
\text { Presencia de violencia de pareja en el embarazo }\end{array}$ & $\begin{array}{c}1 \\
2,689(1,082-6,678)^{\|}\end{array}$ & 0,033 \\
\hline $\begin{array}{l}\text { Adecuada perspectiva espiritual } \\
\text { Deficiente perspectiva espiritual }\end{array}$ & $\begin{array}{c}1 \\
2,503(1,461-4,287)^{q}\end{array}$ & $<0,001$ \\
\hline $\begin{array}{l}\text { Elevada felicidad subjetiva } \\
\text { Reducida felicidad subjetiva }\end{array}$ & $\begin{array}{c}1 \\
2,063(1,214-3,506)^{* *}\end{array}$ & $<0,001$ \\
\hline $\begin{array}{l}\text { Pertenecer a etnia mestiza } \\
\text { Pertenecer a etnia afrodescendiente }\end{array}$ & $\begin{array}{c}1 \\
2,264(0,716-7,160)^{++}\end{array}$ & 0,164 \\
\hline $\begin{array}{l}\text { Sin patología del embarazo } \\
\text { Con patología del embarazo }\end{array}$ & $\begin{array}{c}1 \\
1,447(0,764-2,739)^{\ddagger \ddagger}\end{array}$ & 0,257 \\
\hline $\begin{array}{l}\text { Con apoyo de la familia en el embarazo } \\
\text { Sin apoyo de la familia en el embarazo }\end{array}$ & $\begin{array}{c}1 \\
2,088(0,601-7,257)^{\S \S}\end{array}$ & 0,247 \\
\hline $\begin{array}{l}\text { 49-60 puntos escala perspectiva espiritual } \\
38-48 \text { puntos escala perspectiva espiritual } \\
27-37 \text { puntos escala perspectiva espiritual } \\
16-26 \text { puntos escala perspectiva espiritual } \\
05-15 \text { puntos escala perspectiva espiritual }\end{array}$ & $\begin{array}{c}1 \\
2,864(1,469-5,583)^{\natural} \\
3,603(1,517-8,554)^{\natural} \\
5,518(1,685-18,073)^{\natural} \\
6,053(1,187-30,858)^{\|\| !}\end{array}$ & $\begin{array}{l}0,002 \\
0,004 \\
0,005 \\
0,030\end{array}$ \\
\hline $\begin{array}{l}48,5-60,5 \text { puntos escala familismo } \\
36,5-48,4 \text { puntos escala familismo } \\
24,5-36,4 \text { puntos escala familismo } \\
12,5-24,4 \text { puntos escala familismo } \\
00,5-12,4 \text { puntos escala familismo }\end{array}$ & $\begin{array}{c}1 \\
1,051(0,294-3,760)^{\text {ฯ1 }} \\
3,215(0,851-12,142)^{\text {ฯा }} \\
9,869(1,783-54,622)^{* * *} \\
17,828(3,475-91,525)^{++\dagger}\end{array}$ & $\begin{array}{l}0,939 \\
0,085 \\
0,009 \\
0,001\end{array}$ \\
\hline $\begin{array}{l}23,6-28,0 \text { puntos escala de felicidad } \\
19,1-23,5 \text { puntos escala de felicidad } \\
14,8-19,0 \text { puntos escala de felicidad } \\
06,0-14,7 \text { puntos escala de felicidad }\end{array}$ & $\begin{array}{c}1 \\
1,510(0,805-2,832)^{* *} \\
2,522(1,076-5,914)^{* *} \\
1,413(0,132-15,042)^{* *}\end{array}$ & $\begin{array}{l}0,198 \\
0,033 \\
0,774\end{array}$ \\
\hline
\end{tabular}

(continúa) 
Tabla 3. Factores asociados con el nivel de resiliencia en la regresión logística ajustada. $n=499$ (Continuación)

\begin{tabular}{lcc}
\hline \multicolumn{1}{c}{ Factores } & OR (IC 95 \%) & p* \\
Tercer trimestre de embarazo & 1 & 0,013 \\
Segundo trimestre de embarazo & $0,368(0,167-0,809)^{\S \S \S}$ & 0,808 \\
Primer trimestre de embarazo & $0,917(0,456-1,842)^{\S \S \S}$ & \\
\hline
\end{tabular}

* Valor de p obtenido con la prueba de Wald. † Ajustado por etnia, patologías en el embarazo, apoyo familiar, funcionalidad familiar y edad. ${ }^{\ddagger}$ Ajustado por edad y patologías en el embarazo. ${ }^{5}$ Ajustado por etnia, patologías en el embarazo, puntuación total de la escala de familismo de Bardis y edad. "Ajustado por puntuación total de la escala de perspectiva espiritual y puntuación total de la escala de felicidad subjetiva. " Ajustado por violencia en el embarazo, etnia y edad.

** Ajustado por edad, violencia en el embarazo y patologías en el embarazo. ${ }^{++}$Ajustado por patologías en el embarazo y puntuación total de la escala de perspectiva espiritual. ${ }^{\ddagger}$ Ajustado por etnia, trimestre de embarazo, puntuación total de la escala de familismo de Bardis, apoyo familiar, funcionalidad familiar, violencia en el embarazo, edad, puntuación total de la escala de felicidad subjetiva y nivel autoestima. ${ }^{\S 5}$ Ajustado por puntuación total de la escala de familismo de Bardis y patología en el embarazo. IIII Ajustado por violencia en el embarazo. "Ajustado por patologías en el embarazo y funcionalidad familiar. ${ }^{* * *}$ Ajustado por patología en el embarazo. ${ }^{+\dagger+}$ Ajustado por etnia y funcionalidad familiar. ${ }^{555}$ Ajustado por patologías en el embarazo y edad. Fuente: cuestionario de características sociodemográficas, escala de perspectiva espiritual, escala de familismo de Bardis, APGAR familiar, escala violencia de pareja, escala de felicidad subjetiva, escala de autoestima y escala de resiliencia de Wagnild y Young

\section{DISCUSIÓN}

El nivel de resiliencia es cambiante, varía su presencia según las condiciones del entorno y las circunstancias personales ${ }^{(14)}$. El 12,2 \% de las gestantes adolescentes estudiadas tuvo un NRB, frecuencia inferior a la observada en otra investigación en una población de gestantes adolescentes, con similar región geográfica, en la que se encontró un $34,2 \%{ }^{\left({ }^{9}\right)}$. Ello se puede explicar porque entre las segundas había una mayor proporción de pacientes residentes en áreas rurales y eran de menor nivel socioeconómico. No obstante, por encima de las diferencias en las cifras identificadas, es importante subrayar que varios autores señalan que las personas con el NRB deben ser intervenidas, ya que esa situación conlleva al estancamiento en el desarroIlo psicoafectivo y dificulta la integración social ${ }^{(4,5,13)}$. Se encontró una mayor frecuencia deI NRB entre las gestantes adolescentes afrodescendientes que entre las mestizas. Se podría explicar la diferencia dado que entre las primeras había una mayor cantidad de jóvenes de estratos socioeconómicos más bajos. A menor estrato socioeconómico e inferior nivel educativo es mayor la frecuencia del NRB ${ }^{(4,6)}$. No se identificaron estudios que abordaran el nivel de resiliencia en gestantes adolescentes de acuerdo con consideraciones étnicas o raciales. Las puntuaciones de los variados ítems suelen ser distintos en los diferentes estudios,
Io cual hace posible que se guarde una relación con las características específicas de cada población estudiada ${ }^{(9,13,21)}$.

Las dificultades que tiene que enfrentar una gestante adolescente, si no es capaz de superarlas, podrían llevarla a mayores problemas de salud $y$ a un incremento en la carga de discapacidad ${ }^{(5)}$. Se debe trabajar la resiliencia en las jóvenes embarazadas, ya que la persona resiliente asume menos conductas de riesgo, tiene mejor sociabilización, afianza la autoestima, la autonomía y desarrolla una mayor capacidad para enfrentarse a lo cotidiano ${ }^{(4,6,12)}$. Además, está mejor preparada para afrontar los estímulos estresantes, más abierta a nuevas experiencias, es más flexible ante las demandas de cambio y adquiere una mayor estabilidad emocional ${ }^{(5,13)}$. La resiliencia permite aprovechar las oportunidades, actuar con rapidez y eficacia frente a situaciones amenazantes ${ }^{(12,14,25)}$.

Ahorlu et al., han señalado que una forma efectiva de abordar las amenazas en el marco de los derechos sexuales y reproductivos de las jóvenes y prevenir el embarazo en la adolescencia o enfrentarlo, es fomentar el capital social (relaciones con personas significativas), el económico (dominio sobre los recursos económicos), el cultural (hábitos personales, conocimiento y tradiciones) y el simbólico (honor, reconocimiento y prestigio), ya que todos contribuyen al 
desarrollo integral de las competencias de las adolescentes ${ }^{(10)}$. El embarazo en la adolescencia expone a las jóvenes a riesgos médicos, sociales y económicos $(2,6,10)$. Dado que la resiliencia se debe construir contra una amenaza, Pfeiffer et al., proponen que el aprendizaje, la autoorganización y la creatividad, trabajadas dentro del marco de la "resiliencia reproductiva", son adecuadas para enfrentar los riesgos del embarazo a temprana edad ${ }^{(11)}$.

Una consideración que es importante en Latinoamérica es el familismo ${ }^{(26)}$. En el presente estudio se observó que el deficiente familismo se asoció con un NRB. Varios autores ${ }^{(26-29)}$ sugieren estudiarlo (el familismo) como factor para la resiliencia $y$, además, sostienen que este se debe tomar en cuenta para entender los aspectos inherentes a ella. Bravo et aI. ${ }^{(27)}$, indican que el familismo promueve un ajuste educativo en las madres adolescentes mexicanas que mejora el rendimiento, lo que las lleva a tener un mejor ambiente: más sano y productivo. A su vez, Feldman y Pittman ${ }^{(28)}$, encontraron una asociación entre las expectativas del apoyo familiar y el apego prenatal para tomar mejores decisiones con los cuidados del embarazo. Campos et aI. ${ }^{(29)}$, encontraron que el familismo se asoció, positivamente, con apoyo social y, negativamente, con el estrés o ansiedad durante el embarazo, anotan que el familismo puede ser importante en la resiliencia.

Se encontró, también, que la disfunción familiar fue factor importante asociado al NRB. Se ha señalado que la disfunción familiar repercute negativamente en la salud mental de sus miembros ${ }^{(4)}$, mientras la unión de la familia asegura estabilidad emocional, económica y social ${ }^{(26)}$. Tenezaca et al. ${ }^{(25)}$, en gestantes adolescentes que tuvieron apoyo de sus padres, observaron una menor afección psicológica y un mejor desenvolvimiento socioeducativo; indicaron que el apoyo familiar conlleva a una mejor adaptación, equilibrio emocional $y$ resiliencia. Los adolescentes que pertenecen a familias disfuncionales tienen peor resiliencia ${ }^{(11,13)}$.

La adecuada función familiar aporta soporte emocional, autoestima y permite aprender a reaccionar frente a situaciones adversas. Los integrantes de la familia suelen estar interrelacionados, pues la conducta de uno se verá influenciada por el otro, la respuesta no es lineal (causa-efecto), sino circular, cada familia desarrollará sus propios mecanismos de afrontamiento ${ }^{(4,26)}$

Se observó que la baja autoestima se asoció con cuatro veces el NRB. Ulloque et aI. ${ }^{(9)}$, encontraron una correlación positiva entre autoestima $y$ resiliencia en embarazadas adolescentes, $r h o=0,16$; $p<0,001$. También lo señaló Morales y González ${ }^{(30)}$, en jóvenes de 18 años de edad, rho $=0,51 ; p<0,01$ y, Leiva ${ }^{(31)}$, en adolescentes con vulnerabilidad social, rho $=0,56$; $p<0,01$. La autoestima son los sentimientos de valía personal y de respeto a sí mismo. La persona que presenta alta autoestima suele presentar mayores niveles de resiliencia y será capaz de tener mejor desarrollo de sus competencias personales ${ }^{(30,31)}$.

La violencia de pareja contra la mujer es un problema mundial. Se encontró en el presente estudio que la violencia de pareja, dentro del embarazo, se asoció con dos veces el NRB. Esto lo han observado otros autores ${ }^{(7,12,13,32)}$ que señalan que las mujeres violentadas tienen una menor resiliencia que las que no lo son.

En el estudio se observó que la deficiente perspectiva espiritual fue un factor asociado con el NRB. Duckett et al. ${ }^{(33)}$, lo hicieron en embarazadas adolescentes afroamericanas, observaron que mejor espiritualidad se traducía en mayor resiliencia. Por su parte, Jaramillo-Vélez et aI. ${ }^{(18)}$, en colombianas no gestantes y víctimas de violencia de pareja, encontraron un coeficiente de correlación positiva entre la resiliencia y la espiritualidad ( $r=0,22 ; p=0,0015)$. Las convicciones religiosas o espirituales pueden contribuir a la construcción del sentido de vida, es decir, resiliencia. La relación que establezca el individuo y el ser supremo generará confianza ${ }^{(4)}$. Al respecto, Sa'ad et aI. ${ }^{(34)}$, señalaron que, en embarazadas adolescentes la consejería grupal y la sicología cognitiva Ad-Din centradas en la persona, fueron eficaces para mejorar resiliencia. La sicología cognitiva Ad-Din posee componentes religiosos $y$ espirituales que aportan elementos para fortalecer a las personas que se encuentren en situaciones críticas.

Se encontró que la reducida felicidad subjetiva se asoció con un mayor NRB, lo cual es coherente con lo expuesto por Ulloque et aI. ${ }^{(9)}$, que reportaron una asociación entre la felicidad y resiliencia, indicando que las adolescentes embarazadas que no se sentían 
felices tenían más posibilidades de tener un NRB en comparación con las que se sentían felices, OR 3,7 (IC 95 \%: 1,3-10,3). La infelicidad en el embarazo se relaciona con el bajo peso del neonato al nacer, influye en el comportamiento y el desarrollo infantil ${ }^{(4)}$.

También González-Arratia ${ }^{(14)}$, señala que, en adolescentes existe la correlación positiva moderada entre felicidad $y$ resiliencia. Una persona feliz logra tener éxito en muchos aspectos de la vida, además, Ios que se consideran felices se adaptan y se recuperan mejor de las situaciones traumáticas ${ }^{(4,14)}$.

Se observó que las participantes en el segundo trimestre tuvieron una menor presencia deI NRB, en comparación con las de tercer trimestre: $p=0,013$. En general, en el primer trimestre del embarazo hay cambios negativos del humor y la aparición de sentimientos contradictorios. En el segundo hay mayor felicidad y ánimo, mientras que en el tercero hay ansiedad ante la inminencia del parto ${ }^{(35,36)}$.

Las embarazadas adolescentes estudiadas dijeron las mejores puntuaciones a: "Siento orgullo de haber logrado cosas en mi vida" y "Mi vida tiene sentido"; mientras que entregaron las peores a: "A veces yo hago cosas quiera o no", "Rara vez me preguntó sobre el objetivo de las cosas" y "No insisto en cosas en las que no puedo hacer nada al respecto". No se identificaron otros estudios que abordaran las mismas situaciones, para establecer comparaciones, sin embargo se proponen como temáticas a explorar cuando se busca prevenir o dentro de la atención de las gestantes adolescentes.

La presente evaluación tiene como fortaleza ser uno de los primeros estudios colombianos en estimar la frecuencia deI NRB e identificar las influencias psicosociales en las gestantes adolescentes, enfoque pocas veces adelantado en comunidades latinoamericanas. Además, es de los primeros en explorar la resiliencia, espiritualidad, felicidad, autoestima y el familismo en gestantes adolescentes del Caribe colombiano con escalas mundialmente validadas. Más estudios con diseños diferentes se deben realizar para corroborar los hallazos. Este tiene como limitaciones las propias de los diseños transversales, las asociaciones identificadas no son causales sino estadísticas.

La frecuencia del NRB estimada es propia del grupo involucrado, por tanto, se deben cuidar las extrapolaciones dado que es posible que la muestra no refleje la composición de la población de la cual fue extraída. Sesgos de respuesta y de información, específicamente, en cortesía y memoria, pueden estar presentes.

Todos los sectores de la sociedad deben estar comprometidos en la prevención y en la atención del embarazo en la adolescencia ${ }^{(1-4)}$. Esta problemática no puede seguir mirándose únicamente como un problema obstétrico cuando existen factores socioculturales y económicos fuertemente involucrados. Existen carencias en las acciones de prevención y enfrentamiento del embarazo en la adolescencia.

Es necesario implementar políticas públicas que generen suficiente acompañamiento $y$ apoyo para intervenir multidisciplinariamente a las jóvenes que se convirtieron en madres a temprana edad. Una de ellas es aplicar las estrategias para mejorar la resiliencia ${ }^{(4)}$. Se recomienda a los actores de la atención en la salud explorar el nivel de resiliencia de las gestantes adolescentes, teniendo presente que los factores asociados pueden interactuar en conjunto o por separado ${ }^{(12)}$.

\section{CONCLUSIÓN}

En las gestantes adolescentes de una ciudad del Caribe colombiano que acuden a la consulta prenatal se encontró un NRB: 12,8\%. Familismo, nivel de autoestima bajo, disfunción familiar, violencia de pareja en el embarazo, deficiente perspectiva espiritual y reducida felicidad subjetiva, se asociaron con significancia estadística.

\section{CONFLICTOS DE INTERESES}

Ninguno que declarar.

\section{FINANCIACIÓN}

Proyecto apoyado por la Universidad de Cartagena (Colombia) luego de resultar ganador en la Octava convocatoria interna de proyectos de investigación, Resolución 02565 del 2016, legalizada por medio del acta de compromiso (003-2017) con el grupo de investigación Salud de la mujer. También Colciencias, por medio del Programa Jóvenes Investigadores, apoyó a 
Uiloque-Caamaño y a la logística en la investigación. Los demás investigadores no recibieron honorarios por su participación en el diseño, trabajo de campo, análisis y escritura del documento. Un poster fue presentado en ESG-2019 [XIII Congress of the European Society of Gynecology], Viena, Austria, 2019.

\section{REFERENCIAS BIBLIOGRÁFICAS}

1. UNICEF. The state of the world's children 2011. Adolescence: an age of opportunity [internet]. [Consultado 2019 ago 1]. Disponible en: https://cutt.ly/7e56q9h

2. World Health Organization [internet]. Ginebra: OMS, 2019. [Consultado 2019 ago 1]. Disponible en: https:// cutt.ly/se56iVh

3. Colombia. Ministerio Salud y Protección Social. Encuesta nacional de demografía y salud 2015 (ENDSColombia-2015) [internet]. [Consultado 2019 ago 1]. Disponible en: https://cutt.ly/se56o6Z

4. Monterrosa-Castro A, Ulloque-Caamaño L, ArtetaAcosta C. Estrategias para mejorar el nivel de resiliencia en adolescentes embarazadas. Investig Enferm Imagen Desarr. 2018;20(2):1-12. DOI 10.11144/ Javeriana.ie20-2.emnr.

5. Salazar-Pousada D, Arroyo D, Hidalgo L, Pérez-López FR, Chedraui P. Depressive Symptoms and Resilience among Pregnant Adolescents: A Case-Control Study. Obstet Gynecol Int. 2010;952493. DOI 10.1155/2010/952493.

6. Nordin N, Wahab R, Yunus F. Psychological well-being of young unwed pregnant women: implications for extension education and programs. Procedia Soc Behav Sci. 2012;68:700-709. DOI 10.1016/j.sbspro.2012.12.260.

7. Arteta-Acosta C, Monterrosa-Castro Á, ulloque-Caamaño L. Prevalencia de violencia doméstica Gestacional según el ingreso económico de las naciones. Rev.cienc.biomed. 2016;7(1):80-92.

8. Every Woman Every Chil. Estrategia mundial para la salud de la mujer, el niño y el adolescente (20162030). Sobrevivir, prosperar, transformar [internet]. [Consultado 2019 ago 1]. Disponible en: https://cutt. 1y/De6rVFu

9. Ulloque-Caamaño L, Monterrosa-Castro Á, ArtetaAcosta C. Prevalencia de baja autoestima y nivel de resiliencia bajo, en gestantes adolescentes de poblaciones del caribe colombiano. Rev chil obstet ginecol. 2015;80(6):462-74. DOI 10.4067/S071775262015000600006 .

10. Ahorlu CK, Pfeiffer C, Obrist B. Socio-cultural and economic factors influencing adolescents' resilience against the threat of teenage pregnancy: a cross-sectional survey in Accra, Ghana. Reproductive Health. 2015;12(1):1-11. DOI 10.1186/s12978-015-0113-9.

11. Pfeiffer C, Ahorlu CK, Alba S, Obrist B. Understanding resilience of female adolescents towards teenage pregnancy: a cross-sectional survey in Dar es Salaam, Tanzania. Reproductive Health. 2017:14(77):1-12. DOI 10.1186/s12978-017-0338-X.

12. Cauce AM, Cruz R, Corona M, Conger R. The face of the future: risk and resilience in minority youth. Nebr Symp Motiv. 2011;57:13-32. DOI 10.1007/978-1-44197092-3_2.

13. Johnson KM, Paley FM, Modest AM, Hacker MR, Shaughnessy S, Ricciotti HA, et al. An assessment of depression, psychosocial factors, and resilience among women seeking prenatal care at an urban community health center. Int J Gynaecol Obstet. 2018;140(2):153-8. DOI 10.1002/ijgo.12358.

14. González-Arratia López-Fuentes NI, Valdez-Medina JL. Resiliencia y felicidad: el impacto de hechos vitales negativos y positivos en adolescentes. Pensando Psicol. 2013;9(16):43-53. DOI 10.16925/pe.v9i16.616.

15. Monterrosa-Castro A, Arias-Martínez M. Partos vaginales y cesáreas en adolescentes. Comportamiento entre 1993-2005 Hospital de Maternidad Rafael Calvo, Cartagena. Colombia. Rev Col obstetricia y ginecología. 2007;58(2):107-114.

16. Wagnild GM, Young HM. Development and psychometric evaluation of the Resilience Scale. J Nurs Meas. 1993; 1:165-78.

17. Reed PG. Religiousness among terminally ill and healthy adults. Research in Nursing \& Health. 1986;9(1):35-41. DOI 10.1002/nur.4770090107.

18. Jaramillo-Vélez DE, Ospina-Muñoz DE, Cabarcas-Iğlesias G. Humphreys J. Resiliencia, espiritualidad, aflicción y tácticas de resolución de conflictos en mujeres maltratadas. Rev. Salud Pública. 2005;7(3):281-292. DOI 10.1590/S0124-00642005000300004.

19. Rodríguez M, Fernández M, Pérez M, Noriega R. Espiritualidad variable asociada a la resiliencia. Cuadernos hispanoamericanos de psicología. 2011;11(2):24-49. 
20. Bardis PD. A familism scale. Marriage \& Family Living. 1959;21(4):340-1.

21. Smilkstein G. The patient, the family and the biopsychosocial model. Fam Med. 1988;6:7.

22. McFarlane J, Parker B, Soeken K, Bullock L. Assessing for abuse during pregnancy: severity and frequency of injuries and associated entry into prenatal care. JAMA. 1992;267:3176-3178. DOI 10.1001/ jama.1992.03480230068030.

23. Lyubomirsky S, Lepper HS. A measure of subjective happiness: preliminary reliability and construct validation. Soc Indic Res. 1999;46:137-155. DOI 10.1023/A: 1006824100041

24. Rosenberg $M$. Society and the adolescent self-image. Science. 1965;148(3671):804. DOI 10.1126/science.148.3671.804.

25. Tenezaca R, Fonseca-Vallejo J, Cárdenas-Villamar C. La resiliencia en adolescentes embarazadas, atendidas en el "Hospital Provincial General Docente de la ciudad de Riobamba", periodo 2011-2012. [Trabajo de grado Psicología Clínica]. Riobamba: Facultad de Ciencias de la Salud. Universidad Nacional de Chimborazo; 2012.

26. Monterrosa-Castro A, Ulloque-Caamaño L, Mercado-Lara M, Beltrán-Barrios T. Adolescent Pregnancy: Assessing Familism with Bardis Scale. IJCMR. 2019;6(3):C7-C15. DOI 10.21276/ijcmr.2019.6.3.25.

27. Bravo DY, Umaña-Taylor AJ, Guimond AB, Updegraff KA, Jahromi LB. Family Ethnic Socialization, and Mexican-Origin Adolescent Mothers' Educational Adjustment. Cultur Divers Ethnic Minor Psychol. 2014;20(3):389-400. DOI 10.1037/a0036527.

28. Feldman J, Pittman S. Adolescent pregnancy along the Texas-Mexico border: a systematic analysis of risk and resiliency in a Mexican American population. Social Perspectives. 2008;10(1):29-52.

29. Campos B, Schetter C, Abdou C, Hobel C, Glynn L, Sandman C. Familialism, social support, and stress: positive implications for pregnant Latinas. Cultur Divers Ethnic Minor Psychol. 2008;14(2):155-62. DOI 10.1037/1099-9809.14.2.155.

30. Morales M, González A. Resiliencia-AutoestimaBienestar psicológico y Capacidad intelectual de estudiantes de cuarto medio de buen rendimiento de liceos vulnerables. Estud. pedagóg. 2014;40(1):215-28. DOI 10.4067/S0718-07052014000100013.

31. Leiva L, Pineda M, Encina Y. Autoestima y apoyo social como predictores de la resiliencia en un grupo de adolescentes en vulnerabilidad social. Revista de Psicología. 2013;22(2):111-23. DOI 10.5354/07190581.2013 .30859

32. Lam N, Contreras H, Cuesta F, Mori E, Cordori J, Carrillo N. Resiliencia y apoyo social frente a trastornos depresivos en gestantes sometidas a violencia de género. Rev. peru. Epidemiol. 2008;12(3):1-8.

33. Duckett RJ. Black Adolescent Mothers and their Families: A Phenomenological Study of Resilience. Seton Hall University Dissertations and Theses (ETDs). [Thesis degree Doctor of Philosophy] EE.Uu: Seton Hall; 2009.

34. Sa'ad F, Yusooff F, Nen S, Subhi N. The Effectiveness of person-centered Therapy and Cognitive Psychology Ad-din Group Counseling on Self-concept, Depression and Resilience of Pregnant Out-of-wedlock Teenagers. Procedia Soc Behav Sci. 2014;114:927-32. DOI 10.1016/j.sbspro.2013.12.809.

35. González-Mesa ES, Arroyo-González ML, IbrahimDíez N, Cazorla-Granados O. Mood state at the beginning of the pregnancy and its influence on obstetric and perinatal outcomes. J Psychosom Obstet Gynaecol. 2019;40(2):106-13. DOI 10.1080/0167482X.2018.1427726.

36. Ibrahim SM, Nicoloro-SantaBarbara J, Auerbach MV, Rosenthal L, Kocis C, Et al. Pregnancy-specific coping and changes in emotional distress from mid- to late pregnancy. J Reprod Infant Psychol. 2019;17:1-16. DOI 10.1080/02646838.2019.1578871. 